\title{
Development of a Self-balanced Rock True Triaxial Compression Instrument
}

\author{
Tang Yanchun ${ }^{1}$, Wang Yue ${ }^{1}$, Liu Ruilong ${ }^{1}$, Zhou Hui $^{2}$, Mao Qingfeng ${ }^{1}$ \\ ${ }^{1}$ College of Architecture and Civil Engineering, China Three Gorges University, Yichang, China \\ ${ }^{2}$ Institute of Rock and Soil Mechanics, Chinese Academy of Sciences, Wuhan, China \\ Email address: \\ 126955250@qq.com (Tang Yanchun),332139228@qq.com (Wang Yue)
}

To cite this article:

Tang Yanchun, Wang Yue, Liu Ruilong, Zhou Hui, Mao Qingfeng. Development of a Self-balanced Rock True Triaxial Compression Instrument. American Journal of Civil Engineering. Vol. 7, No. 1, 2019, pp. 9-16. doi: 10.11648/j.ajce.20190701.12

Received: December 25, 2018; Accepted: February 27, 2019; Published: March 8, 2019

\begin{abstract}
Based on the analysis of the structure and the performance of similar instruments at home and abroad, a Self-balanced Rock True Triaxial Compression instrument (SRT) was developed. For SRT, complex stress realization, based on three-dimensional stress alone control and change; and the self-balanced structure is adopted on the basis of frame design, which makes the instrument simple and compact; design of lateral composite press plate with embedded rolling roller, the influence of test results on the effect of friction on the side end surface of the specimen under lateral stress constraint can be eliminate effectiveness; The design scheme of transverse double sliding rod is adopted, its validity can be designed by horizontal alignment, and the problem of eccentric compression of specimen can be partly alleviated in horizontal direction. By changing the alkali of the mothed triaxial chamber, both true triaxial and conventional triaxial compression can be tested. Test results of true triaxial and conventional compression of rocks with different lithology were carried out by SRT. The results, such as the deformation and failure characteristics of rock strength in complex stress conditions, have been obtained by SRT, which indicates that the design of SRT is feasible and feasible. The development of this instrument can promote the further development of Chinese rock triaxial testing machine.
\end{abstract}

Keywords: True Triaxial, Instrument Design, Self-balanced

\section{Introduction}

The rock triaxial compression test machine is one of the main methods of compression test on rock mechanics, including uniaxial compression test, conventional triaxial compression test and true triaxial compression test, etc. The results can be used for studying the basic mechanical characteristics of deep rock mass under complex stress state. At present, at home and abroad, variousfunctions and parameters of rock-triaxial compression testing machine have been designed: The rock uniaxial compression instrument developed by Hudson [1] is mainly used for measuring the uniaxial compression strength of rocks. Through rigid testing machine and electro-hydraulic servo loading, the rock samples can be destroyed stably in the whole process, and the uniaxial compression of rock samples can be obtained with force-strain curve being drawn. However, it can only apply uniaxial stress, and the function of uniaxial compression instrument is simple.
The conventional triaxial compression instrument is used for simulating the triaxial compressive stress of rocks. The stress condition is that the sample is subjected to triaxial stress, in which the axial $\sigma_{1}$ is loaded by rigid medium, and the lateral stress $\left(\sigma_{2}=\sigma_{3}\right)$. The confining pressure is applied by a system through a flexible medium. However, due to the lateral stress $\sigma_{2}=\sigma_{3}$, it is not possible to simulate the stress or failure of deep rock mass, therefore, conventional triaxial compressors can not be used in some situations. For meeting the real needs of engineering and scientific research. The true triaxial compression instrument of rocks is an instrument which can apply triaxial pressure independently. It can simulate three stress conditions and stress paths with different principal stress values. Thus, the RT3-type-rock high-pressure true triaxial instrument developed by Dongjun [2] and Sun Xiaoming [3] and other rocks have developed the nonlinear mechanical test system of true triaxial soft rocks, etc. Rockman 207, developed by Tian Jun [12] et al., can accurately control the rheology of hard rock and meet the 
research needs of short-term and long-term rheology. In terms of the present situation and development trend of true triaxial instrument of deep rock mass and the interpretation of its control system [4], $50 \mathrm{~mm} * 100 \mathrm{~mm}$ and $50 \mathrm{~mm} * 50 \mathrm{~mm} * 100 \mathrm{~mm}$ were applied to test the dimension of triaxial compression samples [7]. The essential difference between true triaxial compressors and conventional triaxial compressors is that the lateral stress $\sigma_{2}$ and $\sigma_{3}$ of true triaxial compressors are applied by two independent systems by using rigid or flexible media, respectively. The compression condition of the sample can be changed according to the actual situation, so the true triaxial compression instrument is more suitable for the deformation and failure of rock mass at complex stress state under the actual engineering conditions. Zhang Xiaowei [13] et al. studied and evaluated the effect of end friction on rock deformation. However, there are some problems which can not be solved. For example, the whole instrument is bulky and its structure is more complicated, the operation difficulty is increased, and the validity of the lateral alignment of the sample in the triaxial chamber is not well guaranteed, the effect of the side-end friction on the lateral stress constraint can not be effectively reduced or even eliminated.

In order to meet the research needs on the basic mechanical properties of deep rock mass, aiming at the structural characteristics of the present true triaxial compression tester for rocks, this paper mainly describes a Self-balanced Rock True Triaxial Compression instrument (SRT), which has its application range due to instrument structure characteristics and shows related test results. It provides an effective test basis for revealing the basic mechanical properties of deep rock mass under complex stress state, and promotes the further development of triaxial test instrument for rocks in China.

\section{Purpose and Main Structure of SRT}

\subsection{Design Purpose and Instrument Requirements}

In order to improve the present situation of true triaxial and satisfy the constraint of triaxial independent pressure, the self-alignment of the sample can eliminate the friction force and meet other multifunctional requirements when the lateral constraint is applied. Therefore, the SRT can be more perfect. The main design requirements of the self-balanced true triaxial compression tester are as follows:

(1) The instrument can independently control and change the three principal stresses: $\sigma_{1}, \sigma_{2}$ and $\sigma_{3}$, with a complex stress state of $\sigma_{1} \geq \sigma_{2} \geq \sigma_{3}$.

(2) The rigid medium is applied to transfer $\sigma_{1}$ and $\sigma_{2}$, and measures are taken to effectively reduce the influence of friction force on the side face of the sample under lateral stress constraint.

(3) The structure design adopts the self-balanced method.

(4) The true triaxial test, and the conventional triaxial compression test can be taken into account.

(5) Servo loading control is applied to control the stress of complex stress paths, the axial and lateral stress-strain relationships are measured, and the data of stress and strain are collected automatically.

\subsection{Instrument Integral Structure}

The SRT consists of a triaxial chamber, a test preprocessing module, a servo control module of high-precision pressure, a deformation measurement module, a collection control system and an oil bypass filter module. The triaxial chamber and the main frame are shown in Figure 1 and 2, respectively.

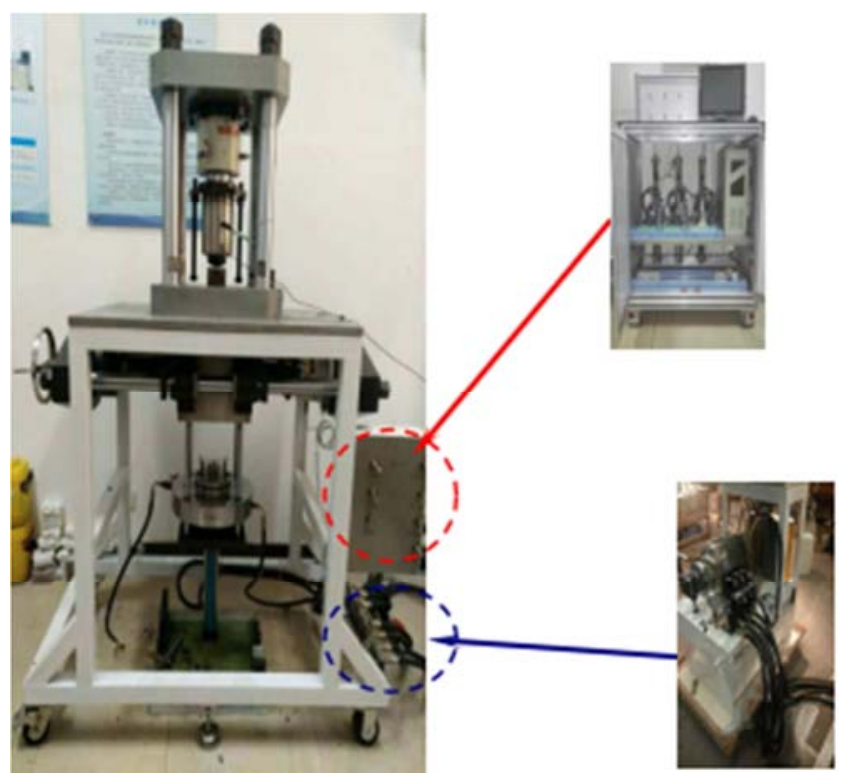

Figure 1. Composition of SRT.

6

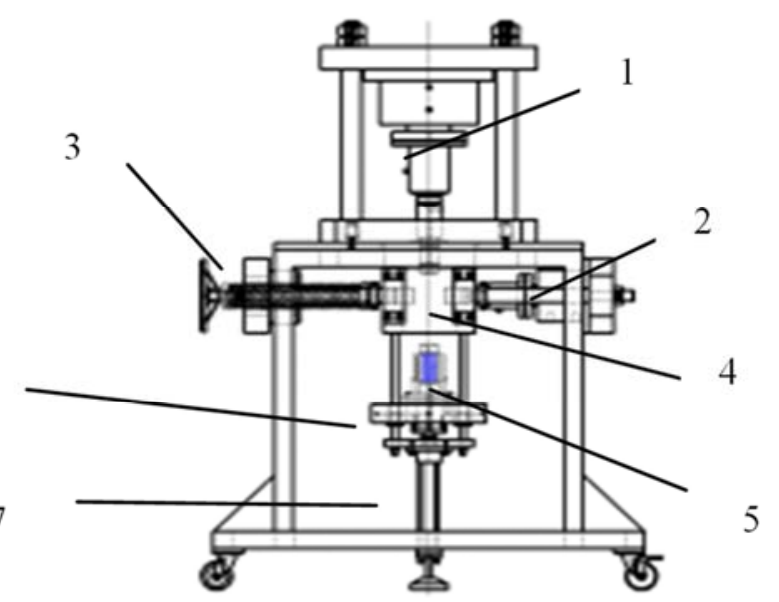

Figure 2. Design of triaxial chamber and main frame of SRT.

Note: $1 . \sigma_{1}$ directional cylinder; 2. $\sigma_{2}$ right side cylinder; $3 . \sigma_{2}$ left helical adjusting unit; 4 . Triaxial chamber; 5 . Sample; 6 . Triaxial chamber base; 7 . Lifting cylinder

\subsubsection{Individual Structural Characteristics of Triaxial Chamber}

First of all, the following size of rock samples should be satisfied: $50 \mathrm{~mm} * 50 \mathrm{~mm} * 100 \mathrm{~mm}$ (the standard sample for true triaxial test) and $50 \mathrm{~mm} * 100 \mathrm{~mm}$ (the standard sample conventional triaxial tests), Secondly, for the pressure index, the maximum axial stress is $1000 \mathrm{KN}$ (for the standard sample, 
the maximum axial stress is $400 \mathrm{MPa}$ ), the lateral maximum $300 \mathrm{KN}$ (for the standard sample, the lateral maximum stress $60 \mathrm{MPa}$ ) and the maximum confining pressure $60 \mathrm{MPa}$ (hydraulic pressure). The purpose of high-strength stainless steel for the final triaxial chamber is to prevent general acid base salt corrosion.

\subsubsection{Characteristics and Application of the Preprocessing}

The preprocessing module is only composed of an oil cylinder. The motor (Figure 3) is the main body of the concentrated load, the stroke of the axial and lateral piston can be controlled more quickly, and a certain amount of prestress is quickly applied. Secondly, the rapid oil filling of the preprocessing module makes the lifting cylinder move, lift or descend the triaxial base, so that the sample can move quickly and steadily in the triaxial compression working area and the preparation area. The lateral stress in $\sigma_{3}$ direction can be restrained by applying flexible medium (hydraulic oil) in triaxial compression test, and a certain confining pressure prestress can be provided.

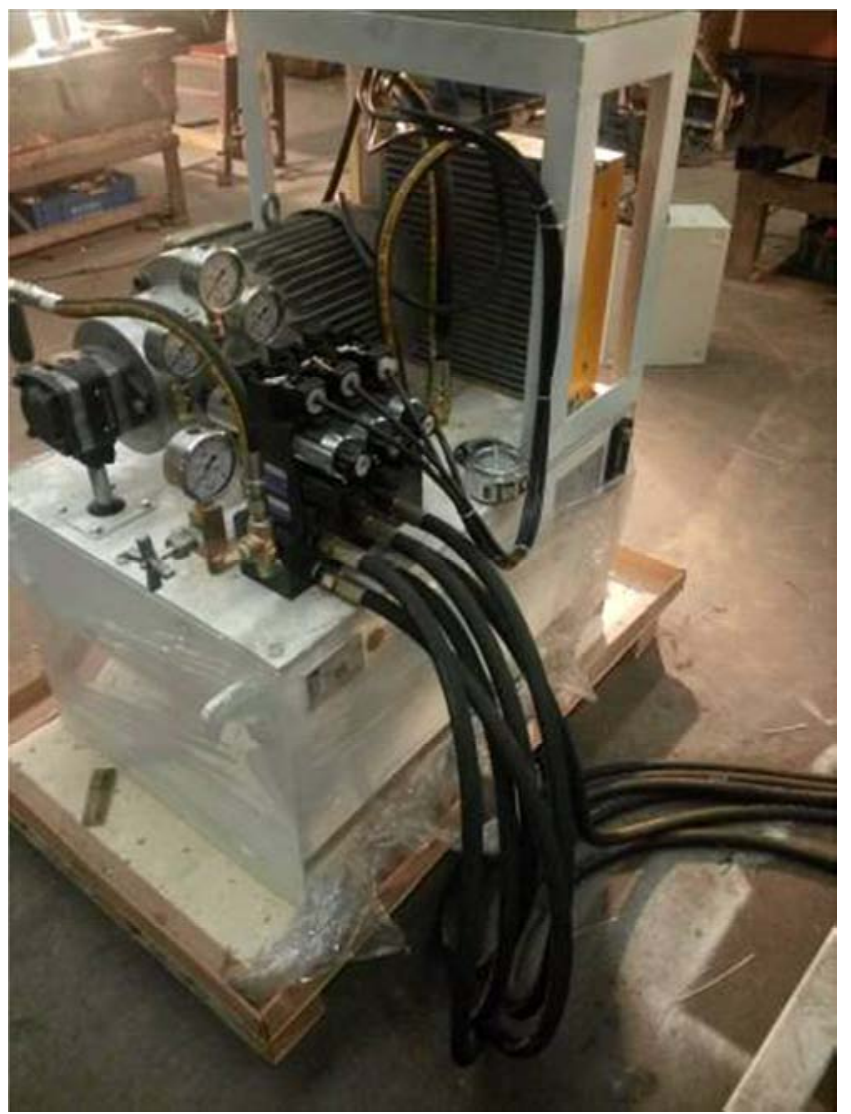

Figure 3. Test preprocessing control instrument.

\subsubsection{High Precision Pressure Servo Control Module}

High precision pressure servo control module can independently provide pressure in three directions (axial pressure, side pressure and confining pressure, and their maximum working pressure can reach $60 \mathrm{MPa}$. The control mode of applied load to the sample can be divided into flow control, step pressure control, constant pressure control, and LVDT displacement control etc. The loading process makes the uniform force fluctuation of the sample extremely small, in which the pressure precision can reach $0.01 \mathrm{MPa}$, and the flow rate is controlled in the range of $0.01-60 \mathrm{mlmin}$. The maximum volume of the pump cylinder under the pressure of $250 \mathrm{ml}$ can be supplied with sufficient pressure to completely destroy the sample, safety protection system-overpressure relief valve protection (non-explosive disc, recyclable) can be applied as the console's safety control system, and pressure protection can be set to avoid overload damage to the instrument.

\subsubsection{Deformation Measurement Module}

(1) The axial displacement in the confining pressure chamber is composed of two LVDT, in which the error range of measurement can reach $\pm 5 \mathrm{~mm}$, the resolution is $0.2 \mu \mathrm{m}$, the maximum working pressure is $60 \mathrm{MPa}$, and the working temperature is $20-95^{\circ} \mathrm{C}$.

(2) The circumferential displacement in the confining pressure chamber is composed of two strain-gauge full bridges (Vishay), the largest working pressure of which is $60 \mathrm{MPa}$, and the working temperature is $20-95^{\circ} \mathrm{C}$.

(3) The displacement of the lateral piston is controlled by one LVDT, and the error range is $\pm 10 \mathrm{~mm}$. The precision of resolution is $0.2 \mu \mathrm{m}$, and the working temperature is $20-95^{\circ} \mathrm{C}$.

\subsubsection{Acquisition Control System}

Using high pressure servo pump, pressure sensor, displacement sensor, industrial control computer to form closed-loop control, accurate control of pressure and displacement, to achieve the set stress path. The PCI (remote sensing image processing) card of 16bit, 16channel, and $100 \mathrm{ks} / \mathrm{s}$ is applied to collect the data of the pressure and displacement sensors, which can adjust the position and interval of the data collected by the software.

\subsubsection{Extended Function}

Multiple channels are reserved for subsequent expansion of acoustic wave, acoustic emission, and other auxiliary detection instrument on the triaxial chamber base.

\subsection{Design Feature of SRT}

\subsubsection{Self-balanced Structure and Application of Complex Stress}

The main frame of the triaxial chamber is designed with self-balanced structure, which is simple, compact and portable. The instrument can be used to study the strength, deformation and failure characteristics of rock under various stress states and stress paths. The available stress state tests include uniaxial compression test, conventional triaxial compression test $\left(\sigma_{1}>\sigma_{2}=\sigma_{3}\right)$, true triaxial compression test $\left(\sigma_{1} \geq \sigma_{2} \geq \sigma_{3}\right)$, and the stress path tests can be carried out with the increase or decrease of confining pressure $\left(\sigma_{2}=\sigma_{3}\right)$ and the increase or decrease of $\sigma_{2}$ and $\sigma_{3}$ alone.

\subsubsection{The Design of the Stress on the End Face of the Specimen}

(1) The principal stress, which has the greatest influence on the mechanical properties of rock, is applied by hydraulic 
pressure, which makes the stress distribution at the end of the sample uniform.

(2) The lateral composite plate (which has embedded rolling roller, as shown in Figure 4) is used to effectively eliminate the influence of the friction force on the side face of the sample under the lateral stress constraint. Lateral compression plate consists of 2. Slotted plate, 3. Rolling roller, 7. Square plate.

(3) Considering the slight difference in the parallelism between the upper and lower sides of the sample along the axial direction, the embedded one is adopted. The spherical ballast with constant radians is loaded on the upper ballast and the stress direction acting on the end face of the sample is fine-tuned to make it consistent with the axial direction.

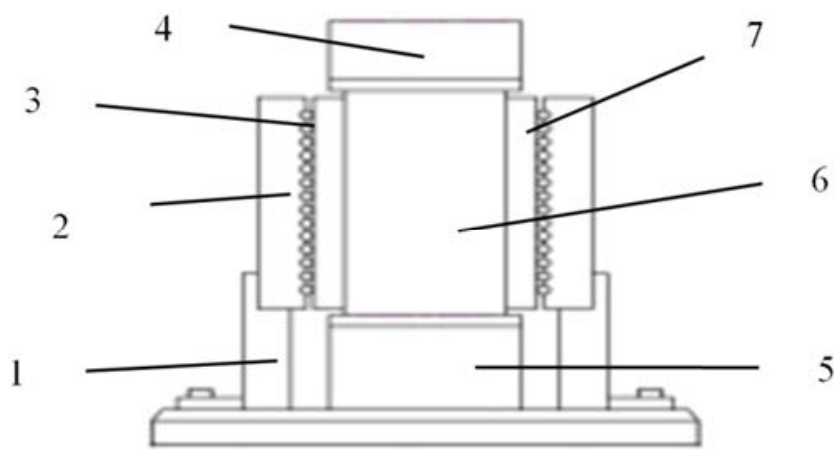

Figure 4. Triaxial chamber structure.

Note: 1. Support seat; 2. Slotted plate; 3 . Rolling roller; 4. Axial compression plate; 5 . Triaxial chamber base; 6 . Sample; 7. Square plate

\subsubsection{Centring Design}

The centring of the sample is designed to take into account the lateral dimension error along the direction of the sample (that is, the error in the lateral alignment of the sample, which will lead to the eccentric compression of the sample in the horizontal direction), adopting the design of the lateral double slide rod (in other words, the left helical adjusting component of the direction is colluded with the hydraulic jack on the right side and the lateral double slider) to maintain the validity of the sample in the lateral pair, remove the eccentric compression of the sample in the horizontal direction. Lateral double slide rod as shown in Figure 5.

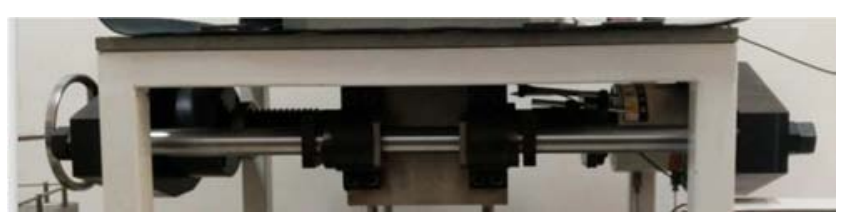

Figure 5. Lateral double slide rod.

\subsubsection{Exchangeable Triaxial Chamber Base}

Considering the true triaxial, the conventional triaxial test adopts the way of nesting and placing the axial base at the center of the triaxial chamber base, so as to realize the purpose of considering the true triaxial and conventional triaxial test. The lower part of the axial base is a cylinder (this part is nested in a triaxial chamber base) and the upper part is a square cylinder coaxial with the cylinder.

\section{Comparison of Technical Indexes and Functions of the Same Type of Instruments}

The main technical indexes and their functions of some representative instruments developed at home and abroad are collected by reading the literature, as shown in Table 1 .

Table 1. Main technical indexes and functions of similar instrument at home and abroad.

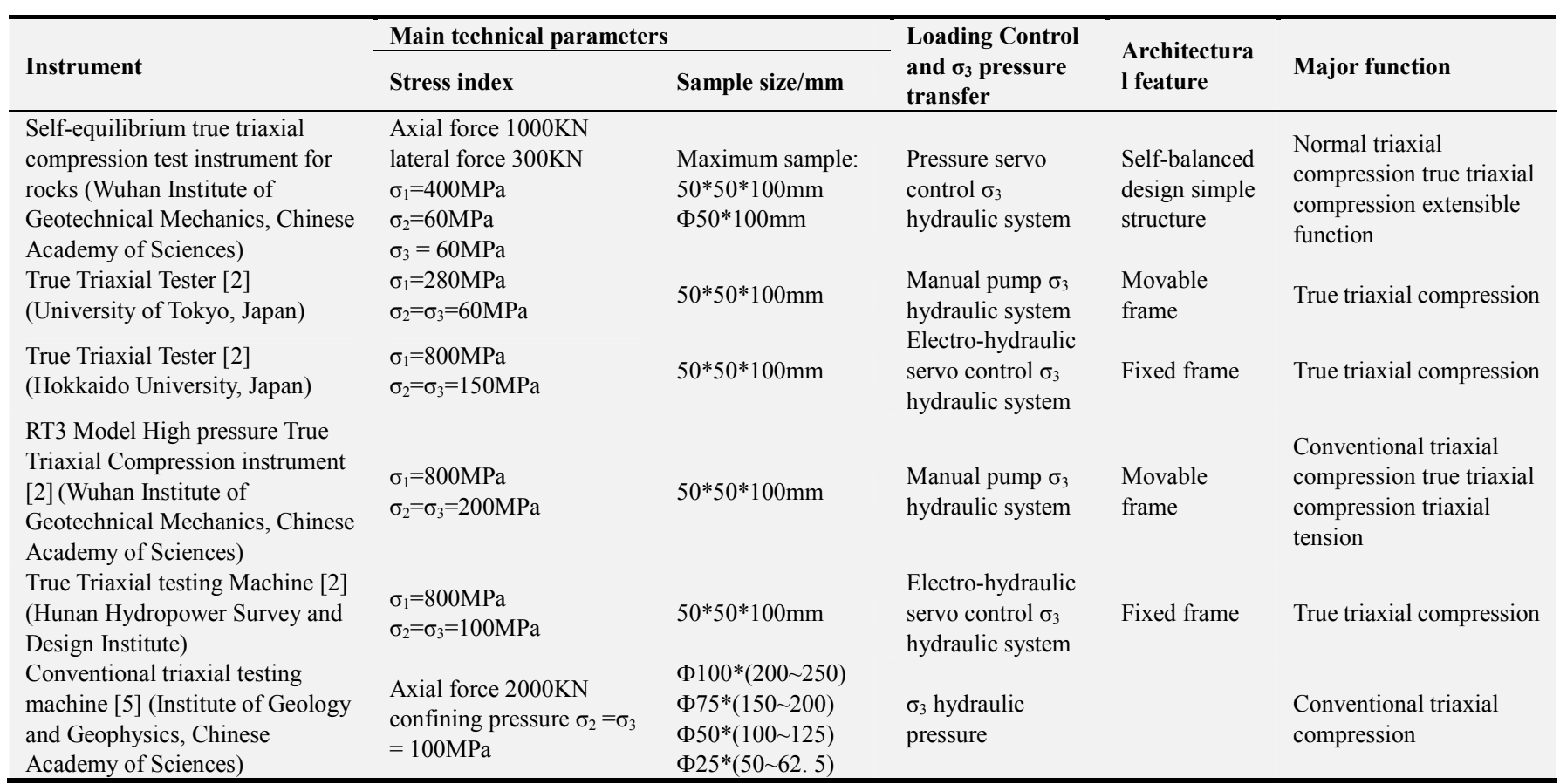




\begin{tabular}{|c|c|c|c|c|c|}
\hline \multirow[b]{2}{*}{ Instrument } & \multicolumn{2}{|l|}{ Main technical parameters } & \multirow{2}{*}{$\begin{array}{l}\text { Loading Control } \\
\text { and } \sigma_{3} \text { pressure } \\
\text { transfer }\end{array}$} & \multirow{2}{*}{$\begin{array}{l}\text { Architectura } \\
\text { I feature }\end{array}$} & \multirow[b]{2}{*}{ Major function } \\
\hline & Stress index & Sample size/mm & & & \\
\hline $\begin{array}{l}\text { True triaxial testing machine [5] } \\
\text { (Central South University) }\end{array}$ & $\begin{array}{l}\text { Axial force } 3000 \mathrm{KN} \\
\sigma_{2} \text { lateral force } 2000 \mathrm{KN} \\
\sigma_{3} \text { lateral force } 2000 \mathrm{KN}\end{array}$ & $\begin{array}{l}\text { Maximum sample: } \\
300 \times 300 \times 300 \mathrm{~mm}\end{array}$ & $\begin{array}{l}\text { Transfer of } \sigma_{3} \\
\text { through rigid } \\
\text { medium }\end{array}$ & $\begin{array}{l}\text { frame } \\
\text { construction }\end{array}$ & True triaxial compression \\
\hline $\begin{array}{l}\text { MTS815 full Digital hydraulic } \\
\text { Servo testing Machine [6] (MTS } \\
\text { Company, USA) }\end{array}$ & $\begin{array}{l}\text { Axial force } 2667 \mathrm{KN} \\
\text { confining pressure } \\
\sigma_{2}=\sigma_{3}=50 \mathrm{MPa}\end{array}$ & $\begin{array}{l}\Phi \\
(50 \sim 100) *(100 \sim 150 \\
) \mathrm{mm}\end{array}$ & $\begin{array}{l}\text { Electro-hydraulic } \\
\text { servo control } \sigma_{3} \\
\text { hydraulic system }\end{array}$ & & $\begin{array}{l}\text { Conventional triaxial } \\
\text { compression }\end{array}$ \\
\hline $\begin{array}{l}\text { TAW-2000 rock triaxial testing } \\
\text { machine (Shandong University of } \\
\text { Science and Technology) }\end{array}$ & $\begin{array}{l}\text { Axial force } 2000 \mathrm{KN} \\
\text { confining pressure } \\
\sigma_{2}=\sigma_{3}=100 \mathrm{MPa}\end{array}$ & $\begin{array}{l}\text { Maximum sample: } \\
\Phi 100 \times 200 \mathrm{~mm}\end{array}$ & $\begin{array}{l}\text { Electro-hydraulic } \\
\text { servo control } \sigma_{3} \\
\text { hydraulic system }\end{array}$ & $\begin{array}{l}\text { door frame } \\
\text { structure } \\
\text { self-balanced } \\
\text { pressure }\end{array}$ & $\begin{array}{l}\text { Conventional triaxial } \\
\text { compression }\end{array}$ \\
\hline $\begin{array}{l}\text { Non-linear mechanical test system } \\
\text { for true triaxial soft rock [3] } \\
\text { (China University of Mining and } \\
\text { Technology) }\end{array}$ & $\begin{array}{l}\text { Maximum pressure } \\
450 \mathrm{KN} \text { maximum tension } \\
75 \mathrm{KN}\end{array}$ & $\begin{array}{l}150 \times 150 \times 150 \mathrm{~mm} \\
100 \times 100 \times 100 \mathrm{~mm}\end{array}$ & $\begin{array}{l}\text { Transfer of } \sigma_{3} \\
\text { through rigid } \\
\text { medium }\end{array}$ & & $\begin{array}{l}\text { Conventional triaxial } \\
\text { compression true triaxial } \\
\text { compression uniaxial } \\
\text { tensile shear test }\end{array}$ \\
\hline
\end{tabular}

In Table 1, the outcome can be obtained as follows:

(1) In terms of stress indicators: $\sigma_{1}=400 \mathrm{MPa}$, with high axial stress index, Li Xinping et al. (2012) studied the distribution law of in-situ stress measured in mainland China. The data show that the maximum horizontal principal stress in mainland China is less than 60MPaat the depth of $500 \mathrm{~m}-2000 \mathrm{~m}$. Although the lateral stress and confining pressure index of this instrument is slightly lower than those of the similar instrument, the design value of $60 \mathrm{MPa}$ basically meets the need of the test research on the basic mechanical properties of deep rock mass. Based on the stress index of this instrument, it can be applied to the test study of the basic mechanical properties of middle hard rocks and middle soft rocks.

(2) In terms of sample size: the sample size is $50 * 50 *$ $100 \mathrm{~mm}$ (true triaxial test sample) and $50 * 100 \mathrm{~mm}$ (conventional triaxial test sample), which meets the standard of most of the same instruments at home and abroad.

(3) On loading control and $\sigma 3$ pressure transfer mode: in the loading control mode, this instrument adopts the pressure servo control mode, which is basically consistent with most similar instruments. Some instruments, such as RT3 high-pressure true triaxial test instrument, use manual pump to control the stress. The main shortcoming of the instrument is that the application and control of stress in three directions need manual operation, thus, it is difficult to ensure synchronism, in $\sigma 3$ transmission mode, the hydraulic pressure is applied in this instrument, which is basically consistent with most similar instruments.

(4) In terms of structural characteristics: in terms of structural characteristics, the instrument adopts self-balanced design. Its structure is simple, and the whole instrument is compact and light. Some instruments adopt fixed frame, movable frame, door frame structure and other structural modes, so that the whole instrument is relatively heavy.

(5) Functional diversification: this instrument can give consideration to conventional triaxial compression, and true triaxial compression test, and can extend the functions of ultrasonic testing, seepage test, and triaxial rheological test.

\section{Analysis on Test Results}

\subsection{Test Methods}

All the true triaxial tests were carried out with the long square column sample of $50 * 50 * 100 \mathrm{~mm}$, and the test was carried out in SRT. The true triaxial standard sample was evenly coated with 704 silicone rubber for the purpose of removing oil, placing the finished sample in the center of the triaxial chamber, clamping the two sides with splint, and covering the axial compression plate over it. First, the motor lifts the sample to the test work area. Then, fix the lifting cylinder, and use the test preprocessing module to carry out the three-way preloading, according to the order of $\sigma_{1}, \sigma_{2}, \sigma_{3}$ (provide a certain pressure value and finish the alignment). The design values are loaded according to the order of $\sigma_{1}, \sigma_{2}$, $\sigma_{3}$, and the preloading is completed for 5 minutes. Then the single direction loading of $\sigma_{1}$ is carried out by the high precision pressure control instrument until the sample is destroyed, and the pressure and axial displacement of the test are recorded by the control instrument in the whole process. The three direction pressure of sample in failure of stopping loading and unloading is carried out in the order of $\sigma_{3}, \sigma_{2}, \sigma_{1}$, and the fracture of the sample is treated with the data of the control instrument. In the conventional triaxial tests, the cylindrical samples of $50 * 100 \mathrm{~mm}$ are used. The hot shrinkage film is applied to close the sample at high temperature, and the upper and lower end face are tightly sealed. As the oil removal treatment, the test process only omits the constraint in $\sigma_{2}$ direction. The oil pressure applied in $\sigma_{3}$ is the same as that of true triaxial test.

The conventional triaxial and true triaxial samples avoid the anisotropy of rock mass to produce a large error in the test, thus, the same large rock mass is used for sample preparation, in which the direction of stratification and lithology of the rock mass is approximately at the ground level [8].

\subsection{Data Analysis}

The true triaxial and conventional triaxial compression tests of rock with different lithology (limestone, basalt) have been carried out by used SRT. The test results obtained are 
described below.

\subsubsection{Conventional Triaxial Compression Test Results of Different Lithology}

According to the test scheme, the conventional triaxial control method is to control $\sigma_{1}$ and change $\sigma_{3}$ only $\sigma_{1}$ is stable to $20 \mathrm{MPa}$, and $\sigma_{3}$ is chosen as four typical variable of $5 \mathrm{MPa}$, $10 \mathrm{MPa}, 15 \mathrm{MPa}, 20 \mathrm{MPa}$, respectively.

The results of conventional triaxial compression tests for different lithology (limestone, basalt) are shown in Table 2.

Table 2. Conventional triaxial test results of different lithology.

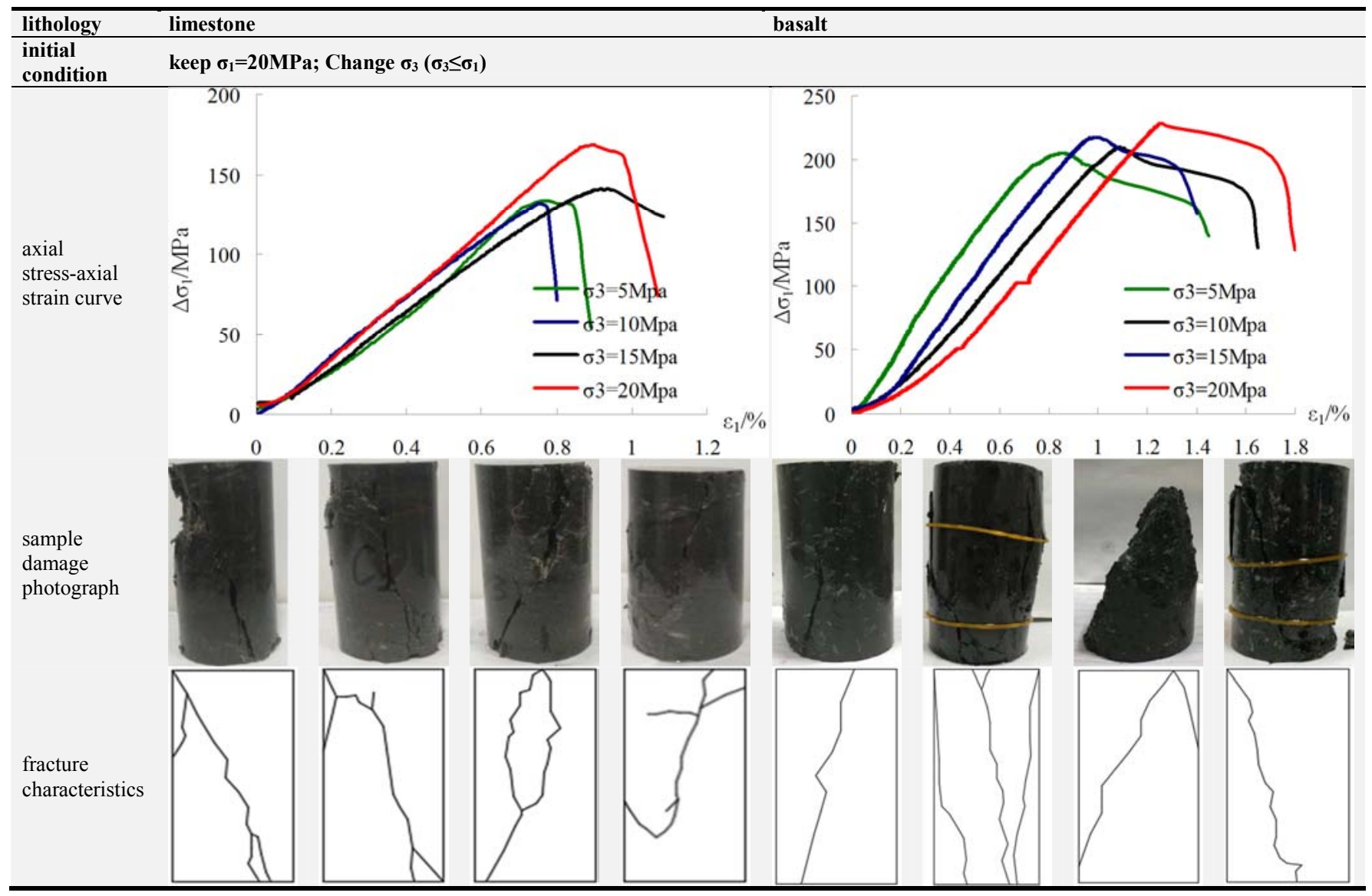

Note: $\Delta \sigma_{1}$ means axial stress increment; $\varepsilon_{1}$ means axial strain

According to the test results in Table 2 the results of conventional triaxial compression test for rocks with different lithology (limestone, basalt) (including their axial stress-axial strain curves) can be obtained. The fracture characteristics basically reflect the deformation and failure characteristics of these two kinds of rock under different confining pressure, which shows that it is feasible to carry out conventional triaxial compression test for rocks with different lithology by means of the self-balanced true triaxial compression test instrument. In the conventional triaxial compression test, the instrument meets the design requirements.

\subsubsection{True Triaxial Compression Test Results of Different Lithology}

According to the test method, two rock samples were tested, and a series of tests were carried out by the method of controlling variables. The typical test data and their phenomena were extracted and analyzed. Limestone: under the condition of initial triaxial stress that $\sigma_{1}=20 \mathrm{MPa}, \sigma_{2}=20 \mathrm{MPa}$, the variables $\sigma_{3}=0 \mathrm{MPa}, \sigma_{3}=10 \mathrm{MPa}$ and $\sigma_{3}=20 \mathrm{MPa}$. Basalt: under the condition that $\sigma_{1}=20 \mathrm{MPa}$, the variables $\sigma_{2}=10 \mathrm{MPa}$ and $\sigma_{3}=0 \mathrm{MPaare}$ in a group, $\sigma_{2}=10 \mathrm{MPa}$ and $\sigma_{3}=10 \mathrm{MPa}$ in a group, and $\sigma_{2}=20 \mathrm{MPa}$ and $\sigma_{3}=10 \mathrm{MPa}$ in a group. The stress-strain curve of the test, the test failure photos and the fracture characteristics are shown in the following table 3. 
Table 3. True triaxial test results of different lithology.

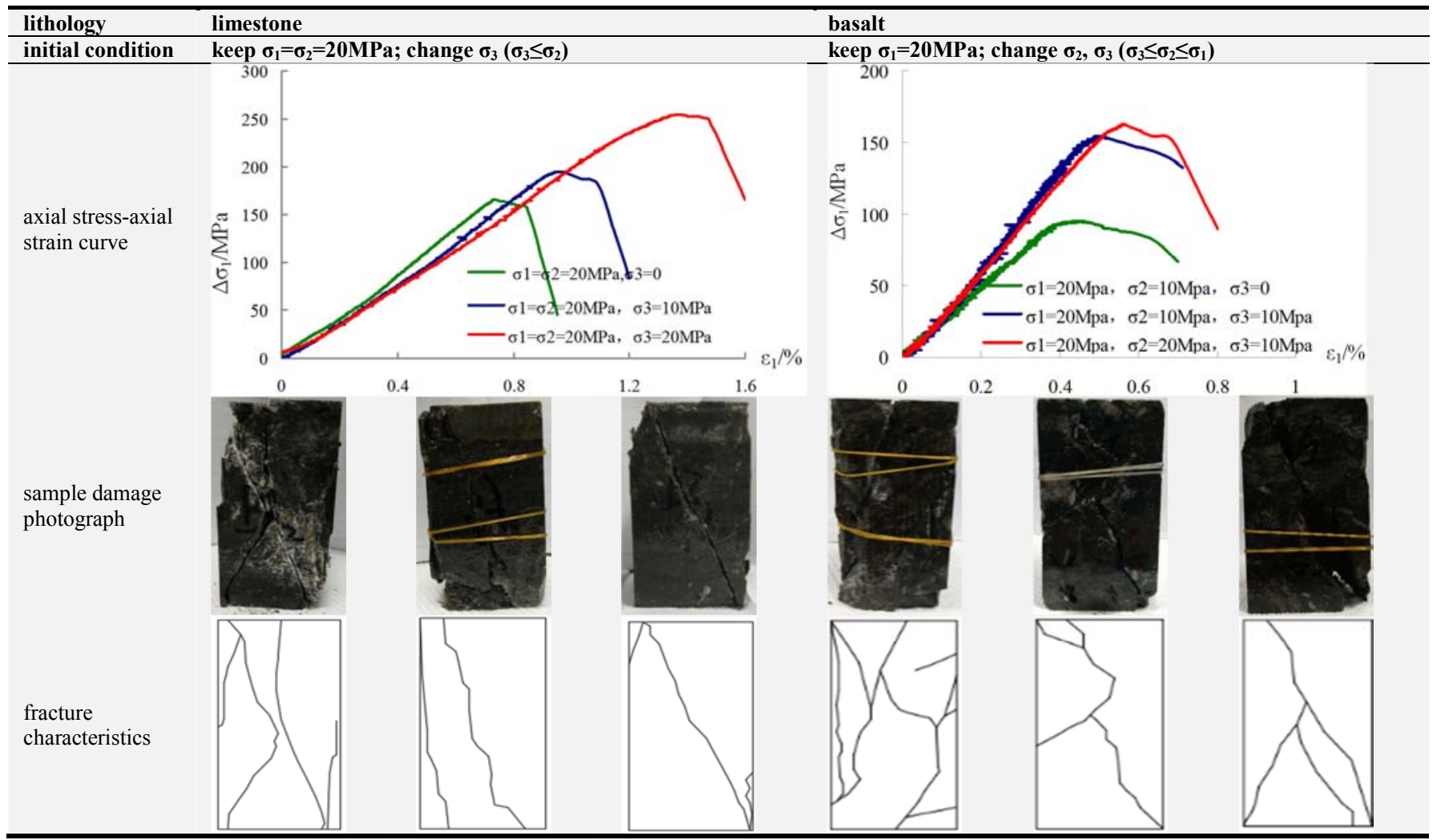

Note: $\Delta \sigma_{1}$ means axial stress increment; $\varepsilon_{1}$ means axial strain

The test results and phenomena of two groups of different lithology under the condition of controlling test variables are given in Table 3. It is obvious that apart from the individual differences of the samples, the increase of $\sigma_{3}$ in triaxial test causes the increase of the peak value, which indicates that the increase of triaxial pressure can increase the peak strength of limestone sample. It can be seen from the test curve that the curve becomes more gentle with the increasing $\sigma_{3}$, which indicates that the strength of the sample has been improved in the elastic-plastic stage after the addition of confining pressure. It can be found from the fracture characteristic diagram that the angle between the fracture and the bottom of the sample is not obvious, but the fracture penetration is better. Based on the above analysis, it can be confirmed that the peak load is a failure load, and the peak strength of limestone sample can be effectively increased by increasing the lateral axial compression properly.

From the results of conventional and true triaxial compression tests (including axial stress-axial strain curves) of different lithology (limestone and basalt), fracture characteristics basically reflect the deformation and failure process of the two kinds of rock from brittle fracture through shear dislocation to plastic flow ${ }^{[9-11]}$. It shows that the deformation and failure characteristics of medium strength rocks (such as limestone and basalt) under complex stress conditions can be obtained by using the SRT. It is feasible to carry out true triaxial compression test for rock with different lithology and meet the design requirements.

\section{Conclusions}

In this paper, a Self-balanced Rock True Triaxial Compression instrument (SRT) was developed. The main conclusions are as follows:

(1) For SRT, the application of complex stress and independently change of three-dimensional stress can be realized; based on self-balanced structural design, simple and compact of triaxial chamber frame structure can be achieved; based on design of lateral composite press plate with embedded rolling roller, the influence of test results which has been produced by friction of the side end surface under lateral stress constraint can be eliminated effectively; through the design of the lateral double slide rod, the effectiveness of the centring scheme can be guaranteed, and the problem of eccentric compression of the sample in the horizontal compression direction can be relieved partially; by the design of the exchangeable triaxial chamber base, both the true triaxial and conventional triaxial compression test can be realized.

(2) By SRT, a series of true triaxial and conventional compression of rocks of different lithology has been carried out. The obtained results, such as deformation and failure characteristics of medium strength rock on complex stress condition, show that design and function of SRT can be satisfied reasonably.

The development of this instrument can promote the further development of rock triaxial compression instrument in 
China.

\section{Acknowledgements}

This paper is supported jointly by National Natural Science Foundation of China (51279089 and 51779129)

\section{References}

[1] Hudson JA, Crouch SL, Fairhurst C. Soft, stiff and servo-controlled testing machines: a review with reference to rock failure [J]. Engineering Geology, 1972, 6 (3): 155-189.

[2] Xu Dongjun, Xing Zhijian, Li Xiaochun, et al. Development of RT3 type rock high pressure true triaxial apparatus [J]. Geomechanics, 1990, 11 (2): 1-14.

[3] Sun Xiaoming, He Manchao, Liu Chengyu, et al. Development of nonlinear mechanical test system for true triaxial soft rock [J]. Journal of Rock Mechanics and Engineering, 2005, 24 (16): 2870-2874.

[4] Li Xinping, Wang Bin, Zhou Guilong. A study on the Distribution of In-situ stress in the mainland of China [J]. Journal of Rock Mechanics and Engineering, 2012, 31 (add. 1): $2875-2880$.

[5] Niu Xuechao, Zhang Qingxi, Yue Chinese. Present situation and development trend of rock triaxial testing machine [J]. Geomechanics, 2013, 34 (2): 600-607.
[6] Tang Haiyan, Li Shulin. MTS815 full digital hydraulic servo testing machine $[\mathrm{J}]$. Research and Development of Mining Industry, 2004, 24 (3): 28-31.

[7] Zhang Ming, Lu Yujie, Yang Qiang. Failure probability and strength size effect of Quasi-brittle Materials [J]. Journal of Rock Mechanics and Engineering, 2010, 29 (9): 1782-1789.

[8] Wu Gang, Sun Jun. Deformation and strength characteristics of fractured rock mass under unloading stress [J]. Journal of Rock Mechanics and Engineering, 1998, 17 (6): 615-621.

[9] He Wenxiu, Zhao Qihua, Zhang Haiquan. Deformation, failure and Acoustic Emission characteristics of basalt under Triaxial Compression [J]. Gansu Water Conservancy and Hydropower Technology, 2015, 51 (05): 26-29.

[10] Wu Shang, Liu Yourong, Li Shijia. Experimental study on mechanical properties and mechanical model of limestone under triaxial compression $[\mathrm{J}]$. Proceedings of the Yangtze River Academy of Sciences, 2013, 30 (03): 30-34.

[11] Tian Yong, Yu ran Gang. Energy Analysis of Limestone triaxial Compression process under different confining pressures [J]. Geomechanics, 2014, 35 (01): 118-122 (129).

[12] Tianjun, Lu Gaoming, Li Yuanhui, Shilei, Ren Jinlai. Full stress-strain curve and rheological test function analysis of hard rock triaxial apparatus [J]. Metal Mine, 2018 (10): 151-156.

[13] Zhang Xiwei, Feng Xiating, Kong Rui, Wang Gang and Peng Shuai. Key technology research on true triaxial apparatus for stress-strain curve of hard rock [J]. Journal of Rock Mechanics and Engineering, 2017, 36 (11): 2629-2640. 